\title{
CONTROL GENÉTICO DE LA PRODUCCIÓN DE POLEN 2n POR "HUSOS PARALELOS" EN PAPAS DIPLOIDES
}

\author{
Elsa L. Camadro; Rodimiro Ortiz; Masaru Iwanaga*
}

\section{RESUMEN}

Se utilizó un diseño de cruzamientos dialélicos para entrecruzar seis clones diploides productores de polen 2 n por "husos paralelos", con amplia base de variabilidad heredable. Las progenies fueron evaluadas por producción de polen $2 \mathrm{n}$ en dos localidades en Perú. El porcentaje de las progenies que produjeron $1 \% \mathrm{o}$ más de polen $2 n$ varió entre 36\% y 86\% en Huancayo y 14\% y 83\% en San Ramón; sin embargo, no se detectaron diferencias estadísticamente significativas entre las 29 familias en las dos localidades. La Aptitud Combinatoria General (ACG), la Aptitud Combinatoria Específica (ACE) y los efectos matemos fueron significativos para el porcentaje de individuos que producían polen $2 \mathrm{n}$ y la frecuencia media de producción de polen $2 n$ por familia, cuando se asumió la existencia de efectos fijos. Los valores de coincidencia de producción de polen $2 n$ en las generaciones de plántula y clonal fueron altos (78\%-83\%).

En cruzamientos recíprocos entre plantas "productoras" y "no productoras" de polen $2 \mathrm{n}$, aproximadamente un cuarto a un tercio de la descendencia produjo este tipo de polen. La producción de polen 2n por "husos paralelos" tendría un control genético relativamente simple. Por ello, el carácter puede manejarse en el mejoramiento genético como si estuviera controlado por un solo gen.

Palabras claves adicionales: restitución de la primera división meiótica, gametos 2n, poliploidización sexual.

Aceptado para publicación: $\quad$ marzo 1,1993

* Ing. Agr, M.S., Ph.D. Científico visitante en el Centro Internacional de la Papa (CIP), Unidad Integrada E.E.A. Balcarce (INTA), FCA (UNMP) y CONICET, C.C. 256 (7620) Balcarce, Argentina; M.S, Ph.D. Dir. actual: IITA, Nigeria; M.S., Ph.D. Dir actual: IBPGR, Via delle Sette Chiese 142, 00145 Roma, Italia 


\section{SUMMARY}

\section{GENETIC CONTROL OF 2n POLLEN PRODUCTION BY "PARALLEL SPINDLES" IN DIPLOID POTATOES}

A diallel mating design was used to intermit six diploid clones with a wide genetic base, that produced $2 \mathrm{n}$ pollen by "parallel spindles". The progenies were evaluated for $2 \mathrm{n}$ |pollen production in two localities in Peru. The percentage of progenies which produced $1 \%$ or more $2 \mathrm{n}$ pollen varied from $36 \%$ to $86 \%$ in Huancayo and from $14 \%$ to $83 \%$ in San Ramón; however, statistically significant differences were not detected for the 29 families in either locality. General Combining Ability (GCA), Specific Combining Ability (SCA) and maternal effects were significant for the percentage of individuals with $2 n$ pollen and the average frequency of $2 \mathrm{n}$ pollen production per family when fixed effects were assumed.

The coincidence values for $2 \mathrm{n}$ pollen production in the seedling and clonal generations was high (78\% to $83 \%$ ). In the reciprocal crosses among $2 \mathrm{n}$ pollen "producer" and "non producer" plants, approximately one-fourth to onethird of the progenies produced $2 \mathrm{n}$ pollen. The production of $2 \mathrm{n}$ pollen by "parallel spindles" is under a relatively simple genetic control. Therefore, the trait can be handled in a breeding program as if it were controlled by a single gene.

Additional Index words: $\quad$ first division restitution, 2n gametes, sexual polyploidization.

En la naturaleza, los poliploides pueden originarse por duplicación espontánea del complemento cromosómico somático (poliploidización asexual) o por el funcionamiento de gametos $2 \mathrm{n}$ (poliploidización sexual). Por definición, los gametos $2 n$ son gametos o gametofitos con el número no reducido de cromosomas, que pueden ser producidos por eventos meióticos anómalos, el desarrollo de células apomeióticas del óvulo, o la duplicación cromosómica pre-o post-meiótica (11,1,5).

En las especies tuberosas silvestres y cultivadas del género Solanum se han observado varias alteraciones en el proceso meiótico que han sido inequívocamente asociadas con la producción de polen $2 n$. Una de ellas consiste 
en la orientación paralela de los husos en anafase II de la microsporogénesis, en contraposición con los husos normales que se orientan en forma tal que sus vértices definen un tetraedro. Los husos paralelos pueden estar fusionados o no.

Algunos investigadores denominan "husos paralelos" tanto a los husos fusionados como a los no fusionados (13) mientras que otros distinguen dos tipos: "husos paralelos" (sin fusionar) y "husos fusionados" $(17,19)$.

Los husos paralelos han sido relacionados con la formación de díadas en el estadio de tetradas, y la producción de polen $2 n$ con núcleos genéticamente equivalentes a aquellos formados por restitución de la primera división meiótica (polen $2 \mathrm{n}$ por RPD)(2).

Existe aún controversia entre diferentes investigadores con respecto a la relación entre la presencia de anormalidades en los husos y la existencia de disturbios meióticos (15,6,8,13,17). Mok y Peloquin (13) y Veilleux y col. (19) han sugerido que "husos paralelos", "husos fusionados" y "husos tripolares" (que dan como resultado una tríada de dos microsporos $\mathrm{n}$ y uno $2 \mathrm{n}$ ) podrían simplemente representar niveles de intensidad en la expresión de un mismo carácter.

Para poder explotar el potencial ofrecido por los gametos 2n con núcleos genéticamente equivalentes a aquellos formados por restitución de la primera división meiótica en el mejoramiento genético, es importante conocer la herencia del (los) mecanismo(s) citológico(s) que llevan a la formación de este tipo de gametos.

Los datos genéticos obtenidos por Mok y Peloquin $(13,14)$ con seis clones diploides de origen híbrido (Solanum tuberosum Grupo Phureja x haploide de $S$. tuberosum Grupo Tuberosum) dan sustento a la hipótesis de que la orientación paralela de los husos en anafase II está controlada por un gen mutante recesivo con penetrancia incompleta. Resultados similares han sido informados en alfalfa (10).

Existen indicaciones, sin embargo, de que el fondo genético y el ambiente pueden influenciar la expresión de la capacidad para producir polen 2n por RPD.

Veilleux y Lauer (18) observaron que en S. tuberosum Grupo Phureja la expresión relativamente constante o variable de la producción de polen $2 \mathrm{n}$ por RPD dependía del genotipo particular que se estaba investigando. Los genotipos constantes tenían el mismo nivel de expresión del carácter, independientemente del ambiente en el que eran cultivados. En los genotipos variables, por otro lado, se observaron diferencias en la producción de polen $2 \mathrm{n}$ en lóculos, anteras y botones florales de una misma planta (19), lo que es indicativo de diferencias microambientales.

La temperatura (4) y el fotoperíodo (16) también afectan la frecuencia de polen 2n producido por un genotipo. 
Jacobsen (7) cultivó plantas de un clon diploide en diferentes lugares de un invernáculo e informó que, en un muestreo, esas plantas produjeron $0.4 \%$ a $31.0 \%$ de polen de tamaño grande. Este investigador postuló que la herencia del carácter "producción de polen $2 \mathrm{n}$ " sigue un patrón poligénico, y que algunas progenies superan a la media parental.

En general, los estudios sobre la herencia de la producción de polen 2n por RPD han sido realizados con pocos genotipos de estrecha base genética. Para obtener más información sobre la herencia de este carácter, se realizó un análisis dialélico con progenitores seleccionados en poblaciones con germoplasma silvestre y cultivado, que producían polen 2n por el mecanismo de "husos paralelos".

\section{MATERIALES Y MÉTODOS}

Se utilizó un diseño de cruzamientos dialélicos que no incluyó las autofecundaciones (Método Experimental 3, de acuerdo a Griffing (3)) para entrecruzar seis clones diploides de origen híbrido. Estos clones habían sido seleccionados por resistencia a agentes bióticos y abióticos adversos, y producción de polen 2n por el mecanismo de "husos paralelos" (Tabla 1).

Las semillas resultantes de los cruzamientos fueron puestas a germinar en bandejas. Las plántulas de tres semanas fueron trasplantadas en macetas Jiffy-7 y llevadas al campo cuatro semanas más tarde.

Las evaluaciones se realizaron en dos localidades en Perú: Huancayo

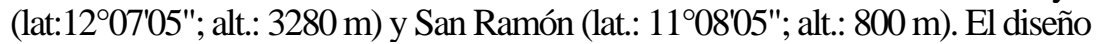
experimental utilizado en ambas localidades fue el de Bloques Completos Aleatorizados (BCA), con tres repeticiones de 30 plantas cada uno.

En floración, se tomaron muestras de polen que se colorearon sobre portaobjetos con carmín acético en glicerol (9) y se observaron con microscopio óptico. Las plantas que produjeron polen de tamaño heterogéneo, con $1 \%$ o más de polen de tamaño grande, fueron clasificadas como "productoras" de polen $2 \mathrm{n}$.

Diez genotipos por familia, en las tres repeticiones, se tamizaron por producción de polen $2 \mathrm{n}$ dos veces en un período de tres semanas. Estos genotipos se cultivaron como clones en Huancayo y se evaluaron durante un segundo año.

Una muestra de las progenies del cruzamiento dialélico, clasificadas como "productoras" (P) y "no productoras" (NP) de polen 2n se utilizaron como progenitores en cruzamientos recíprocos "P-NP" y "P-P". La descendencia de estos cruzamientos fue evaluada en dos localidades, Huancayo, Perú, y Balcarce (lat.: $\left.37^{\circ} 45^{\prime} 05^{\prime \prime}\right)$, Argentina. 
Tabla 1. Fuentes de germoplasma, reacción a agentes bióticos y abióticos adversos y frecuencia de producción de polen $2 n$ en seis clones diploides utilizados en el análisis dialélico.

\begin{tabular}{|c|c|c|c|}
\hline Clon & $\begin{array}{c}\text { Fuente de } \\
\text { germoplasma }^{1}\end{array}$ & $\begin{array}{c}\text { Reacción a agentes } \\
\text { bióticos y abióticos adversos }\end{array}$ & $\begin{array}{c}\text { Frecuencia de } \\
\text { polen } 2 n^{2}\end{array}$ \\
\hline 381328.18 & $\begin{array}{l}\text {.phu-stn } \\
\text {.sps }\end{array}$ & $\begin{array}{l}\text { Resistente al nematodo } \\
\text { del nudo }\end{array}$ & $\mathrm{M}$ \\
\hline 381348.7 & $\begin{array}{l}\text {.phu-stn } \\
. s p s \\
. t b r\end{array}$ & $\begin{array}{l}\text { Resistente al nematodo } \\
\text { del nudo }\end{array}$ & A \\
\hline FH-122 & .phu-stn & Resistente al tizón temprano & M \\
\hline FH-92 & .phu-stn & Tolerante al calor & B \\
\hline MI-49.10 & $\begin{array}{l}. t b r \\
. a d g \\
. s p s \\
. c h c \\
. p h u\end{array}$ & $\begin{array}{l}\text { Resistente a la marchitez } \\
\text { bacteriana }\end{array}$ & A \\
\hline MI-17.12 & $\begin{array}{l}. a d g \\
. s p s \\
. p h u\end{array}$ & $\begin{array}{l}\text { Resistente a la marchitez } \\
\text { bacteriana }\end{array}$ & B \\
\hline
\end{tabular}

'phu=phureja; sth = stenotomun;sps=sparsipilum; tbr=tuberosum; adg=andigena; chc $=$ chacoense, ${ }^{2} \mathrm{~B}=$ baja $(1-10 \%) \mathrm{M}=$ media $(11-29 \%) ; \mathrm{A}=$ alta $(>30 \%)$

\section{RESULTADOS}

En la Tabla 2 se presentan los resultados del tamizado por producción de polen $2 n$ de las progenies generadas por el diseño de cruzamientos dialélicos. El porcentaje de las progenies que produjeron $1 \%$ de polen $2 \mathrm{n}$ o más varió entre $36 \%$ y 86\% en Huancayo y entre 14\% y 83\% en San Ramón. El porcentaje de individuos que produjeron polen 2n fue más alto en Huancayo (59\%) que en San Ramón (51\%); sin embargo, no se detectaron diferencias estadísticamente significativas entre las 29 familias.

Los valores de Aptitud Combinatoria General (ACG), Aptitud Combinatoria Específica (ACE) y efectos matemos fueron estadísticamente significativos para 
el porcentaje de individuos que producían polen $2 \mathrm{n}$ y la frecuencia media de producción de polen $2 \mathrm{n}$ por familia, cuando se asumió la existencia de efectos fijos.

Tabla 2. Porcentaje de plantas productoras de polen 2n en la descendencia de 29 familias cultivadas en Huancayo y San Ramón, Perú.

\begin{tabular}{|c|c|c|c|c|c|c|c|}
\hline \multicolumn{8}{|c|}{ Machos } \\
\hline Hembras & 381328.18 & 381348.7 & FH-122 & FH-92 & MI-49.10 & MI-17.12 & $\begin{array}{l}\text { Hembra } \\
\text { media }\end{array}$ \\
\hline \multirow[t]{3}{*}{381328.17} & $\ldots$ & $71.0^{1}$ & 6.0 & 48.0 & 69.0 & 68.0 & 64.5 \\
\hline & $\ldots$ & $83.0^{2}$ & 14.0 & 44.0 & 30.0 & 67.0 & 47.6 \\
\hline & $\ldots$. & $77.0^{3}$ & 40.0 & 46.0 & 49.5 & 67.5 & 56.0 \\
\hline \multirow[t]{3}{*}{381348.7} & 72.0 & $\ldots$. & 66.0 & 66.0 & 70.0 & 86.0 & 72.2 \\
\hline & 7.0 & $\ldots$. & 62.0 & 67.0 & 31.0 & 52.0 & 55.8 \\
\hline & 69.5 & .... & 64.0 & 66.5 & 50.5 & 69.0 & 63.9 \\
\hline \multirow[t]{3}{*}{ FH-122 } & 36.0 & 61.0 & .... & 53.0 & 71.0 & 54.0 & 54.8 \\
\hline & 67.0 & 56.0 & $\ldots$ & 26.0 & 45.0 & 80.0 & 54.8 \\
\hline & 51.5 & 58.5 & .... & 39.5 & 58.0 & 67.0 & 54.9 \\
\hline \multirow[t]{3}{*}{ FH-92 } & 59.0 & 52.0 & 59.0 & .... & 58.0 & n.a. & 57.0 \\
\hline & 46.0 & 61.0 & 45.0 & $\ldots$. & 36.0 & n.a. & 47.0 \\
\hline & 52.5 & 56.5 & 52.0 & $\ldots .$. & 47.0 & n.a. & 52.0 \\
\hline \multirow[t]{3}{*}{ MI-49.10 } & 61.0 & 54.0 & 62.0 & 65.0 & $\ldots$. & 56.0 & 60.0 \\
\hline & 60.0 & 56.0 & 65.0 & 47.0 & $\ldots$. & 22.0 & 50.0 \\
\hline & 60.5 & 55.0 & 63.5 & 56.0 & $\ldots$. & 39.0 & 54.8 \\
\hline \multirow[t]{3}{*}{ MI-17.12 } & 44.0 & 55.0 & 42.0 & 39.0 & 44.0 & $\ldots$. & 45.0 \\
\hline & 53.0 & 35.0 & 47.0 & 28.0 & 48.0 & $\ldots$. & 42.2 \\
\hline & 48.5 & 45.0 & 44.5 & 33.5 & 46.0 & $\ldots$. & 43.5 \\
\hline \multirow[t]{3}{*}{ Macho medio } & 54.5 & 58.6 & 59.3 & 54.1 & 62.1 & 66.3 & $\ldots$. \\
\hline & 58.6 & 58.2 & 46.6 & 42.4 & 38.0 & 55.2 & $\ldots$. \\
\hline & 56.5 & 58.4 & 52.8 & 48.3 & 50.3 & 60.6 & .... \\
\hline \multirow[t]{3}{*}{ Progenitor } & 59.5 & 65.4 & 57.1 & 55.4 & 61.0 & 54.4 & 58.9 \\
\hline & 53.1 & 57.0 & 50.7 & 44.4 & 44.0 & 48.0 & 49.7 \\
\hline & 56.3 & 61.2 & 53.9 & 49.9 & 52.5 & 52.1 & 54.3 \\
\hline
\end{tabular}

${ }^{1}$ Huancayo; ${ }^{2}$ San Ramón; ${ }^{3}$ Huancayo y San Ramón 
Los valores de coincidencia (expresados en porcentaje) para producción de polen $2 \mathrm{n}$ en dos muestras que se obtuvieron en plantas cultivadas a partir de semilla botánica (generación de plántula) y una muestra de la primera generación clonal se presentan en la Tabla 3. Para los datos agrupados, el porcentaje de coincidencia entre las dos muestras tomadas en la generación de plántulas fue 83\% y el de las muestras tomadas en la generación de plántulas y en la primera generación clonal fue $78 \%$. Once por ciento de los individuos que produjeron polen $2 \mathrm{n}$ en la generación de plántula produjeron sólo polen $\mathrm{n}$ en la primera generación clonal. Un porcentaje similar de plantas clasificadas como "no productoras" de polen 2n durante la generación de plántula produjeron polen $2 \mathrm{n}$ en la generación clonal.

Tabla 3. Coincidencia (en porcentaje) de producción de polen $2 n$ en genotipos muestreados durante las generaciones de plántula y clonal.

\begin{tabular}{|c|c|c|c|c|c|}
\hline \multicolumn{6}{|c|}{ Machos } \\
\hline Hembras & 381328.18 & 381348.7 & FH-122 & FH-92 & MI-49.10 \\
\hline \multirow[t]{3}{*}{381328.18} & .... & $80^{1}$ & 100 & 90 & 100 \\
\hline & $\ldots .$. & $78^{2}$ & 88 & 91 & 100 \\
\hline & $\ldots$. & $100^{3}$ & 88 & 100 & 100 \\
\hline \multirow[t]{3}{*}{381348.7} & 90 & $\ldots$ & 90 & 67 & 80 \\
\hline & 82 & $\ldots$ & 75 & 67 & 80 \\
\hline & 70 & $\ldots$ & 80 & 100 & 100 \\
\hline \multirow[t]{3}{*}{ FH-122 } & 90 & 88 & $\ldots$ & 70 & 90 \\
\hline & 50 & 100 & .... & 50 & 60 \\
\hline & 64 & 86 & $\ldots$ & 40 & 50 \\
\hline \multirow[t]{3}{*}{ FH-92 } & 90 & 100 & 50 & $\ldots$ & 89 \\
\hline & 60 & 57 & 78 & $\ldots .$. & 70 \\
\hline & 80 & 33 & 77 & $\ldots$. & 89 \\
\hline \multirow[t]{3}{*}{ MM9.10 } & 70 & 100 & 54 & 75 & $\ldots$ \\
\hline & 73 & 90 & 90 & 75 & $\ldots .$. \\
\hline & 100 & 80 & 80 & 75 & $\ldots$ \\
\hline
\end{tabular}

Momento de muestreo (generaciones): ${ }^{1} 1$ ra de plántula-2da de plántula;

${ }^{2} 1$ ra de plántula-clonal; ${ }^{3} 2$ da de plántula-clonal

En los cruzamientos recíprocos entre las plantas "productoras" y "no productoras" de polen 2n, aproximadamente un cuarto a un tercio de la descendencia produjo polen $2 n$ dependiendo, respectivamente, de si ninguno de los progenitores producían polen 2n o sólo uno de ellos producía este tipo de polen (Tabla 4). 
Tabla 4. Número de plantas productoras de polen $2 n$ en la descendencia de cruzamientos entre plantas "productoras" (P) y "no productoras" (NP) de polen $2 n$.

\begin{tabular}{ccccc}
\hline $\begin{array}{l}\text { Tipo de } \\
\text { cruzamiento }\end{array}$ & $\begin{array}{l}\mathrm{N}^{\circ} \text { de } \\
\text { Familias }\end{array}$ & $\mathrm{N}^{\circ}$ de plantas & $\begin{array}{l}\mathrm{N}^{\circ} \text { de plantas } \\
\text { c/polen 2n }\end{array}$ & $\begin{array}{l}\text { \% de plantas } \\
\text { c/polen 2n }\end{array}$ \\
\hline PxNP & 6 & 146 & 56 & 38.0 \\
NPxP & 4 & 91 & 30 & 32.0 \\
NPxNP & 22 & 788 & 214 & 27.5 \\
\hline
\end{tabular}

\section{DISCUSIÓN}

Mendiburu y Peloquin (11) fueron los primeros en reconocer el valor de los gametos $2 \mathrm{n}$ formados por RPD en el mejoramiento genético. Estos investigadores observaron respuestas fuertemente heteróticas en progenies tetraploides derivadas de cruzamientos $4 \mathrm{x} \times 2 \mathrm{x}$ : estas respuestas fueron atribuidas al mecanismo genético único por el cual se forman gametos diplandroides con núcleos genéticamente equivalentes a los formados por restitución de la primera división meiótica.

Para poder utilizar el polen 2n como herramienta en los programas de mejoramiento genético, es necesario que la producción de este tipo de polen esté bajo control genético y sea, por ello, un fenómeno recurrente. En todas las familias generadas mediante cruzamientos entre plantas que producían polen $2 \mathrm{n}$ a través del mecanismo "husos paralelos" se observaron, en frecuencias intermedias, plantas con este tipo de polen. Además, fue posible obtener con facilidad plantas productoras de polen $2 \mathrm{n}$ mediante cruzamientos aun entre plantas no productoras de estas familias. Todo ello permite sugerir que el mecanismo citológico de producción de polen $2 \mathrm{n}$ tiene un control genético relativamente simple. Sin embargo, los valores estadísticamente significativos de la ACE y los efectos maternos indican que la expresión de la capacidad para producir polen 2n está influenciada por el fondo genético y el ambiente interno, respectivamente.

Los valores de coincidencia obtenidos para la producción de polen 2n de cada familia fueron variables, pero relativamente altos en la mayoría de los casos. La variabilidad observada puede ser tomada como una indicación de las influencias ambientales en la expresión del (los) determinante(s) genéticos de este carácter. Debido a estas influencias, es difícil realizar mediciones precisas de las frecuencias máximas de producción de polen 2 n de distintos genotipos a menos que, como señalaran Owen y col. (16), todos ellos tengan respuestas similares al ambiente en el cual se los cultiva. 
Para poder aumentar la eficiencia de los cruzamientos 4x x 2x en la generación de tetraploides es necesario obtener más información sobre la influencia de los factores ambientales en el proceso meiótico. Pero aun cuando se desconozcan los mecanismos precisos de herencia de la capacidad para producir polen 2n, es posible transmitir este carácter entre genotipos en forma relativamente sencilla mediante cruzamientos. Como McCoy (10) observara en alfalfa, este carácter puede ser manejado en los programas de mejoramiento genético como si estuviera controlado por un solo gen.

\section{REFERENCIAS BIBLIOGRÁFICAS}

1. Asker, S. 1980. Gametophytic apomixis. Elements and genetic regulation. Hereditas 93:277-293.

2. Camadro, E.L. 1986. Los gametos 2n en el origen y la evolución de las angiospermas poliploides. Mendeliana 7 (2): 85-100.

3. Griffing, B. 1956. Concept of general and specific combining ability in relation to diallel crossing systems. Austr. J. Biol. Sci. 9: 463-493.

4. Haynes, K.G; Haynes, F.L.; Swallow, W.H. 1987. Variability of flowering and $2 \mathrm{n}$ pollen production in diploid potatoes under high temperatures. Am.Potato 1.64:35-40.

5. Hermsen, J.G. TH. 1984. Mechanisms and genetic implications of 2n gamete formation. Iowa State J. Res. 58 (4): 421-434.

6. Hoglund, M. 1970. Meiosis in Solanum phureja. Hereditas 66: 183-188.

7. Jacobsen, E. 1976. Cytological studies of diplandroid production in a dihaploid potato clone and its correlation with seed set in $4 \mathrm{x}-2 \mathrm{x}$ crosses. Z. Pflanzenzuchtg 77:10-15.

8. Lam, S.L. 1974. Origin and formation of unreduced gametes in the potato. J.Hered. 65: 175-178.

9. Marks, G.E. 1954. An aceto-carmine glycerol jelly for use in pollen fertility counts. Stain Tech. 29: 277.

10. McCoy, T. J. 1982. The inheritance of $2 \mathrm{n}$ pollen formation in diploid alfalfa Medicago sativa. Can J. Genet. Cytol. 24: 315-323. 
11. Mendiburu, A.O.; Peloquin, S.J. 1976. Sexual polyploidization and depolyploidization: some terminology and definitions. Theor. Appl. Genet. 48:137-143.

12. Mendiburu, A.O.; Peloquin, S.J. 1977. The significance of 2n gametes in potato breeding. Theor. Appl. Genet. 49:53-61.

13. Mok, D. W.S.; Peloquin, S.J. 1975a. Three mechanisms of 2n pollen formation in diploid potatoes. Can. J. Genet. Cytol. 17: 217-225.

14. Mok, D.W.S.; Peloquin, S.J. 1975b. The inheritance of three mechanisms of diplandroid (2n pollen formation) in diploid potatoes. Heredity 35 (3): 295-302.

15. Müntzing, A. 1933. Studies on meiosis in diploid and triploid Solanum tuberosum L. Hereditas 17:223-245.

16. Owen, H.R.; Veilleux, RE.; Haynes, F.L.; Haynes, K.G. 1988. Photoperiod effects on $2 \mathrm{n}$ pollen production, response to anther culture, and net photosynthesis of a diplandrous clone of Solanum phureja. Am. Potato J. 65:131-139.

17. Ramanna, M.S. 1979. A re-examination of the mechanisms of $2 \mathrm{n}$ gamete formation in potato and its implications for breeding. Euphytica 28: 537-559.

18. Veilleux, R.E.; Lauer,F. I. 1981. Variation of 2n pollen production in clones of Solanum phureja Juz. andBuk. Theor. Appl. Genet. 59: 95100.

19. Veilleux, R.E.; McHale, N. A.; Lauer, F.I. 1982. 2n gametes in diploid Solanum: frequency and types of spindle abnormalities. Can. J. Genet. Cytol. 24: 301-314 